\title{
Eye Movements in English Lexical Ambiguity Resolution
}

\author{
Huijun Tang ${ }^{1,2}$ \\ ${ }^{1}$ College of International Studies, Southwest University, Chongqing, China \\ ${ }^{2}$ College of Foreign Languages and Culture, Chengdu University, Chengdu, China \\ Correspondence: Huijun Tang, College of International Studies, Southwest University, Chongqing, China. E-mail: \\ helenaccnu@hotmail.com
}

Received: November 12, 2017 Accepted: December 3, 2017 Online Published: December 23, 2017

doi:10.5539/ijel.v8n2p148 URL: http://doi.org/10.5539/ijel.v8n2p148

\begin{abstract}
English Language ambiguity has always been one of the major concerns of psycholinguistic research. A number of studies have been carried out to investigate different types of ambiguity resolution, including phonological ambiguity resolution, lexical ambiguity resolution, syntactic ambiguity resolution and pragmatic ambiguity resolution. Studies of lexical ambiguity resolution have found that lexically ambiguous words with two meanings of the same frequency took longer to read than lexically unambiguous words, suggesting that different meanings of ambiguous words compete for integration to the local context. This paper gives an overview of the classical psycholinguistic studies on English lexical ambiguity resolution which employed the eye-tracking technology.
\end{abstract}

Keywords: lexical ambiguity, language processing, eye movements

\section{Introduction}

A serial of psycholinguistic studies have investigated lexical ambiguity resolution and discovered that ambiguous lexically items which had two meanings that are equally frequent took longer to read than lexically unambiguous words, suggesting that a mechanism of competition of different meanings of ambiguous words was involved in processing. Rayner \& Duffy (1986) observed that when the two meaning of an ambiguous word are similar in frequency, the reading times for that ambiguous word was longer, compared to its unambiguous control word. It suggests that two equally frequent meanings of an ambiguous lexical item were both activated at the same stage of processing. Therefore, there could be competition of the two meanings during lexical access and also the integration to the context was energy-consuming as both the meanings were equally plausible as input. The same pattern of effect was found in later studies of Rayner and colleges. In Duffy, Morris, \& Rayner (1988), the results showed that the reading times for the ambiguous words with two equally frequent meaning were longer than the unambiguous words. This paper gives a general introduction to the development and major focus of eye movement research in reading comprehension and reviews a serial studies by famous American psycholinguist Keith Rayner and colleges investigating issues of lexical ambiguity resolution.

\section{Eye Tracking and Ambiguity Resolution}

\subsection{Eye Movement Research in Reading}

Reading is an important cognitive process of human being and the earliest study of eye movements in reading can be traced back to the late 19th century. The study of eye movements discovered that peoples' visual line do not move forward constantly, but instead jump from one fixation to another. The movement of jumping from one fixation stage to another is called saccade. McConkie \& Rayner (1975) have pioneered the research of eye movements and their initial research focus was on how eye movements were controlled during reading. Rayner's early studies provided solid empirical footing to Huey's early analysis of reading.

\subsection{Models of Ambiguity Resolution}

There are different opinions about the mechanisms underlying ambiguity resolution. There are two competing accounts in research on ambiguity. According to one account, a mechanism of competition is involved in ambiguity resolution which results in ambiguous words or sentences being harder to understand than unambiguous words or sentences. However, the other account assumes that only one analysis is initially activated in sentence processing and reanalysis occurs only when the initial analysis is found to be implausible. 
According to this reanalysis-based account, sentences processing are easy when there are ambiguities, compared to sentences without ambiguity.

According to the competition model, the processor activates two or more analyses at the same time when encountering ambiguity and these analyses compete with each other. The competition can be very strong when the analyses are activated equally. This occurs when linguistic information supports two or more analyses to a similar extent. Competition model assumes that it is hard for the language processor to process the sentence when there is an ambiguity. This is because when there is an ambiguity, the processor has to activate two or more interpretations. These analyses compete because either of the interpretations could be applied to the ambiguous words or sentences. By contrast, contexts can be very easy to process when there are unambiguous words and sentences because only one interpretation is highly activated by the various sources of information and hence the interpretations do not compete.

In contrast to the competition model, reanalysis model claims that in ambiguity resolution the processor initially adopts one analysis and then reanalyses if the initial analysis is found to be impossible or implausible. According to these models, ambiguous words or structures can make sentence processing very easy because the processor adopts one interpretation first and does not need to go back for reanalysis as long as the interpretation is plausible. However, sentences with unambiguous words or structures are comparatively harder to process because the processor may initially adopt an implausible analysis and would therefore need to go back for reanalysis when the implausibility is discovered.

\section{Eye-movement Research in Lexical Ambiguity Resolution}

\subsection{Word Frequency and Lexical Access}

Rayner \& Duffy (1986) carried out two eye-movement experiments and found that the lexical access time was not significantly affected by verb complexity but it might be affected by the meaning frequency of the word. When the two meanings of a word were equally frequent, it may affect the access of the word and post-access integration.

In the first experiment, they used sentences like "She thought the punch (cider) was a little sour." with the ambiguous word "punch" paired with an unambiguous word "cider". Each ambiguous word had two noun meanings (e.g., punch) and the unambiguous control word only had one meaning (e.g., cider), which is similar to the non-dominant meaning of the target ambiguous word. They assumed that increased fixation time on target words would occur if lexical ambiguity caused immediate processing difficulties and that this increased fixation time might reflect an increase in lexical access time, post-access integration time, or both. This is also consistent with competition, assuming that the lexical ambiguity caused competition between both possible interpretations resulting in an increased fixation time. They analysed fixation durations on the target ambiguous word and also one word that was fixated before the target word and one word after the target word. No significant effect of lexical ambiguity was found for the gaze duration eye movement measure and first fixation duration measure. However, subsequent analyses suggested that the lack of effect of ambiguity may result from the fact that there were a number of lexical items whose two meanings were not equally biased. Two meanings of an ambiguous word may only increase the difficulties of processing when they are equally frequent (i.e., the ambiguous word is equibiased). Indeed, there was longer mean gaze duration $(269 \mathrm{msec})$ for the ambiguous words that were equibiased than ambiguous words that were non-equibiased $(236 \mathrm{msec})$. Furthermore, the mean total time of the ambiguous target words $(314 \mathrm{msec})$ was significantly longer than of the unambiguous control words $(262 \mathrm{msec})$ and the reading times for the disambiguating region were longer in the ambiguous target word condition (82 $\mathrm{msec})$ than in the unambiguous target word condition $(64 \mathrm{msec})$.

\subsection{Word Bias and Lexical Ambiguity Resolution}

\subsubsection{Research Design}

In a serial of eye-movement experiments, the relationship of word bias and lexical processing has been investigated. In the second experiment of Rayner \& Duffy (1986), the experimenters used sentences with equibiased ambiguous lexical items (the ambiguous word had two equally frequent meanings) or non-equibiased lexical items (the ambiguous word had one strongly dominant meaning). Each equibiased ambiguous word (e.g., coach in (1a)) and non-equibiased ambiguous word (e.g., perch in (1b)) was paired with an appropriate unambiguous control word (e.g., cabin in (1a) and trout in (1b)). The disambiguating information always followed the target words.

1a) He found the coach (cabin) was too hot to sleep in.

1b) He saw the perch (trout) had avoided the hook. 
They assumed that it would be more difficult to process equibiased ambiguous words than non-equibiased ambiguous words because it should be more time-consuming to choose from two similar-frequency meanings than from two different-frequency meanings.

In Duffy, Morris, \& Rayner (1988), they used sentences which contained two clauses: the target clause and the disambiguating clause (e.g., "Unfortunately his case (face) was ignored although it had a unique, weathered appearance."). The ambiguous words they used were either equibiased words or non-equibiased words and each ambiguous word was paired with an unambiguous control word. In half of the sentences, the disambiguating clause preceded the target word while in the other half it followed the target word (Before condition and After condition, respectively). When the target word was a non-equibiased ambiguous word, the disambiguating information always supports the subordinate (less frequent) meaning.

Mechanisms of lexical ambiguity resolutions in reading were investigated by Rayner \& Frazier (1989) by conducting two eye-movement experiments using sentences frames like:

2a) George said that the wire informed John that his aunt would arrive on Monday.

2b) George said that the wire surrounded the entire barracks including the rifle range.

2c) George said that the wire was supposed to inform John that his aunt would arrive on Monday.

2d) George said that the wire was supposed to surround the entire barrack including the rifle range.

Two versions of the sentences were disambiguated in accordance with the dominant interpretation of the target word "wire" ( $2 \mathrm{a} \& 2 \mathrm{c})$, and two were consistent with the subordinate interpretation of the target word ( $2 \mathrm{~b} \& 2 \mathrm{~d})$. Two versions contained a disambiguating word (inform or surround, in the example) immediately following the ambiguous target word ( $2 \mathrm{a} \& 2 \mathrm{~b}$ ), and the other two versions neutral material (three or four words 0 were inserted between the ambiguous word and the disambiguating word ( $2 \mathrm{c} \& 2 \mathrm{~d}$ ). The ambiguous word either had two almost equally likely meanings (nonbiased) or had a strong dominant meaning (biased).

\subsubsection{Results and Analysis}

According to their experimental data of Rayner \& Duffy (1986), subjects spent extra time looking at ambiguous target words as compared to their control words when the two meanings of the ambiguous words were equally frequent. However, for the non-equibiased ambiguous target words, when the two meanings were different in frequency, there were no such time differences. Therefore they claimed that both meanings were accessed for the equibiased ambiguous target words but only the dominant meaning was accessed for the non-equibiased ambiguous target words. In other words, competition exists when processing equibiased ambiguous words because the two meanings of the ambiguous word had similar frequencies making it harder to process and more time-consuming. However, no competition should exist in non-equibiased ambiguous words condition as only the dominant meaning was accessed.

Duffy, Morris, \& Rayner (1988) replicated the finding of Rayner \& Duffy's (1986). Measures of processing time for three regions were calculated: the target word (e.g., caset/face), the post-target region (e.g., was ignored) and the disambiguating region (e.g., although it had a unique, weathered appearance). For the target word region, the results in the disambiguating After condition showed that reading times on equibiased ambiguous targets were longer than the unambiguous control words but the reading times of non-equibiased ambiguous targets were no longer than their controls. The results in the disambiguating Before condition was just in contrast. The non-equibiased ambiguous words showed processing difficulty while the equibiased target words did not take more time than their controls. It suggested that the in the disambiguating Before condition of the items with non-equibiased ambiguous targets, the disambiguating context makes the subordinate meaning of the ambiguous word more accessible. Therefore, both the appropriate and inappropriate meanings become available simultaneously and the competition between these two meanings causes processing difficulty. Similar processing difficulty occurred for the equibiased ambiguous words in the After condition when both meanings of the ambiguous target word were accessed at the same time and the two analyses competed with each other. This was consistent with the lengthened reading time on the ambiguous targets.

What's more, the results of Rayner \& Frazier (1989) showed that readers spent more time on the nonbiased target ambiguous words than on the biased target words. This replicated the findings reported in Rayner \& Duffy (1986) and Duffy et al. (1988) who claimed that an ambiguous word was hard to process when it had two almost equally frequent meanings, compared to when it had one dominant meaning. It suggests that the two interpretations of the nonbiased ambiguous word compete and hence results in longer fixation times on the ambiguous word. However when the ambiguous word is biased, the most likely meaning was made available as soon as the word was encountered and was integrated with the prior text before the less likely meaning became 
available. Hence, the competition of the two meanings was avoided.

\section{Discussion}

In all reviewed studies (Duffy, Morris, \& Rayner, 1988; Rayner \& Duffy, 1986; Rayner \& Frazier, 1989), the researchers agree in general that readers always access more than one meaning of a lexical ambiguous word when the ambiguous word is equibiased. The fixation time for ambiguous words was longer than unambiguous words, providing that there was no disambiguating information preceding the target words. This indicated that the activation of two or more meanings of ambiguous words causes competition in processing and hence ambiguous words are harder than unambiguous words to comprehend. As one important part of psycholinguistic studies on English language ambiguity resolution, these studies have provided solid foundation of the Competition model which claims that ambiguous pronoun resolution involves competition between the different interpretations (e.g., Gernsbacher, 1989; MacDonald \& McWhinney, 1995). The idea is that if a pronoun has more than one possible antecedent, the activation levels of the different interpretations are similar, and therefore, it takes a long time before one of the interpretations reaches critical threshold activation and/or the alternative interpretations are sufficiently inhibited. By contrast, if a pronoun is unambiguous, there is no time-consuming competition, so unambiguous pronouns are predicted to be easier to process than ambiguous pronouns.

These results are quite different from the results from the studies investigating syntactic ambiguity resolution, which argues for a process of "reanalysis" rather than "competition" during processing. They claim that in ambiguity resolution the processor initially adopts one analysis and then reanalyses if the initial analysis is found to be impossible or implausible. According to these models, ambiguous words or structures can make sentence processing very easy because the processor adopts one interpretation first and does not need to go back for reanalysis as long as the interpretation is plausible. However, sentences with unambiguous words or structures are comparatively harder to process because the processor may initially adopt an implausible analysis and would therefore need to go back for reanalysis when the implausibility is discovered.

\section{Conclusion}

Eye movements tracking is an effect way of investigating people's processing mechanism in reading activities and it helped to draw a clear picture of various types of ambiguity resolution. The eye movement research in lexical ambiguity resolution showed that language processors are involved in a process of competition between different interpretations of the ambiguous lexical item. The competition lengthened the fixation time and hence brought forward processing difficulties. Further eye movement studies on lexical ambiguity resolution could focus on the processing mechanisms on different stages of processing and discover the interconnection between different types of ambiguity resolutions in reading.

\section{Acknowledgments}

This research is funded by the Sichuan Provincial Social Science Planning Project: SC15WY001.

\section{References}

Duffy, S. A., Morris, R. K., \& Rayner, K. (1986). Lexical Ambiguity and fixation times in reading: Effects of word frequency, verb complexity, and lexical ambiguity. Memory \& Cognition, 14(3), 191-201. https://doi.org/10.1016/0749-596X(88)90066-6

Duffy, S. A., Morris, R. K., \& Rayner, K. (1988). Lexical Ambiguity and fixation times in reading. Journal of Memory and Language, 27, 429-446. https://doi.org/10.1016/0749-596X(88)90066-6

Garnham, A., Oakhill, J., \& Cruttenden, H. (1992). The Role of Implicit Causality and Gender Cue in the Interpretation of Pronouns. Language and Cognitive Processes, 7(3/4), 231-255. http://dx.doi.org/10.1080/01690969208409386

Garnham, A., Oakhill, J., Ehrlich, M., \& Carreirs, M. (1995). Representation and processes in the Interpretation of pronouns: New evidence from Spanish and French. Journal of Memory and Language, 34, 41-62. https://doi.org/10.1006/jmla.1995.1003

Gernsbacher, M. A. (1989). Mechanisms that improve referential access. Cognition, 32, 99-156. https://doi.org/10.1016/0010-0277(89)90001-2

MacDonald, J. L., \& MacWhinney, B. (1995). The time course of anaphor resolution: effects of implicit verb causality and gender. Journal of Memory and Language, 34, 543-566. https://doi.org/10.1006/jmla.1995.1025 
MacDonald, M. C., \& MacWhinney, B. (1990). Measuring inhibition and facilitation from pronouns. Journal of Memory and Language, 29, 469-492. https://doi.org/10.1016/0749-596X(90)90067-A

McConkie, G. W., \& Rayner, K. (1975). The span of the effective stimulus during a fixation in reading. Perception \& Psychophysics, 17(6), 578-586. https://doi.org/10.3758/BF03203972

Rayner, K., \& Duffy, S. A. (1986). Lexical complexity and fixation times in reading: Effects of word frequency, verb complexity, and lexical ambiguity. Memory and Cognition, 14, 191-201. https://doi.org/10.3758/BF03197692

Rayner, K., \& Frazier, L. (1989). Selection mechanisms in reading lexically ambiguous words. Journal of Experimental Psychology Learning, Memory, and Cognition, 15, 779-790. https://doi.org/10.1037//0278-7393.15.5.779

Rigalleau, F., \& Caplan, D. (2000). Effects of gender marking in pronominal coindexation. The Quarterly Journal of Experimental Psychology, 53A(1), 23-52. https://doi.org/10.1080/713755884

Van Gompel, R. P. G., Pickering, M. J., \& Traxler, M. J. (2001). Reanalysis in sentence processing: Evidence against current constraint-based two-stage models. Journal of Memory and Language, 45, 225-258. https://doi.org/10.1006/jmla.2001.2773

Van Gompel, R. P. G., Pickering, M. J., Pearson, J., \& Liversedge, S. P. (2005). Evidence against competition during syntactic ambiguity resolution. Journal of Memory and Language, 52, 284-307. https://doi.org/10.1016/j.jml.2004.11.003

Vonk, W. (1984). Pronoun comprehension. In A. G. Gale \& F. Jognson (Eds.), Theoretical and Applied Aspects of Eye Movement Research. Amsterdam: Elsevier Science Publisher B. V. (North-Holland).

Vonk, W. (1985). On the purpose of reading and the immediacy of processing pronouns. In R. Groner, G. W. McConkie, \& C. Menz (Eds.), Eye Movements and Human Information Processing. Amsterdam: North-Holland.

\section{Copyrights}

Copyright for this article is retained by the author(s), with first publication rights granted to the journal.

This is an open-access article distributed under the terms and conditions of the Creative Commons Attribution license (http://creativecommons.org/licenses/by/4.0/). 